\title{
Psychopathology, Anxiety or Attentional Control: Determining the Variables Which Predict IRAP Performance
}

\author{
Emma Nicholson • Aife Hopkins-Doyle • \\ Dermot Barnes-Holmes • Richard A. P. Roche
}

Published online: 9 April 2014

(C) Association of Behavior Analysis International 2014

\begin{abstract}
Background \& Objectives The Implicit Relational Assessment Procedure (IRAP) is a widely used measure of implicit cognition which has detected biases pertaining to various psychological constructs from spider fear to cocaine dependence. One issue which emerges in the IRAP literature is that of participants failing to meet or uphold the necessary criteria to complete the IRAP, which results in their elimination from the study, which can be rather detrimental to results. The present study sought to delineate which factors may contribute to high attrition rates in IRAP research.

Methodology A previously used IRAP measuring spider fear was employed along with an $n$-back task and a series of questionnaires.

Results Results indicated that the ability to inhibit prepotent responses and focus attention on the task at hand was the best predictor of accuracy on the IRAP, irrespective of spider fear or current levels of anxiety.

Conclusions These results are promising for the use of the IRAP in clinically-relevant domains as they suggest that the psychopathology of participants does not affect performance on the IRAP.
\end{abstract}

The Implicit Relational Assessment Procedure (IRAP; BarnesHolmes et al. 2006) is a widely used measure of so-called implicit cognition. It is a computer-based latency-focused measure which requires participants to respond quickly and accurately to sets of stimuli in a way that is consistent or inconsistent with their previously established responses biases (BarnesHolmes et al. 2010a). The IRAP was derived from a behavioranalytic account of language and cognition called Relational

E. Nicholson $(\bowtie) \cdot$ A. Hopkins-Doyle $\cdot$ D. Barnes-Holmes R. A. P. Roche

Department of Psychology, National University of Ireland,

Maynooth, Co. Kildare, Ireland

e-mail: Emma.Nicholson@nuim.ie
Frame Theory (RFT; Hayes et al. 2001). The fundamental assumption of RFT is that the fundamental components of human language and cognition are relational, and, thus, the IRAP focuses on assessing relations between stimuli (e.g., Hughes et al. 2011). The basic hypothesis of the IRAP is that average response latencies for a group should be shorter across blocks deemed to be consistent relative to inconsistent trials (Barnes-Holmes et al. 2010a), with the assumption that the degree to which the consistent trials differ from the inconsistent trials provides an index of the strength of the verbal or relational responses being assessed (Barnes-Holmes et al. 2010a, b).

Numerous studies have been carried out which demonstrate the basic IRAP effect that participants will respond faster on trials that are consistent with their previously established verbal relations than those that are inconsistent. An account of this effect has been offered in the form of the Relational Elaboration and Coherence Model (REC; BarnesHolmes et al. 2010a) which posits that particular IRAP trials produce brief and immediate relational responses (BIRR's) before the participant presses a response key. The likelihood of these BIRR's occurring will be determined by the verbal history of the participant and current contextual variables. These studies encompass a wide variety of domains including those that are relevant to the assessment of psychopathology, socially sensitive topics such as race and issues around methodology. The IRAP has successfully detected spider fear (Nicholson and Barnes-Holmes 2012a), attitudes toward meat and vegetables exhibited by meat-eaters and vegetarians (Barnes-Holmes et al. 2010b), self-esteem (Vahey et al. 2009), disgust in relation to obsessive-compulsive tendencies (Nicholson and Barnes-Holmes 2012b), cocaine dependence (Carpenter et al. 2012), sexual attraction to children among sexual offenders (Dawson et al. 2009) and depression (Hussey and Barnes-Holmes 2012). Critically, it appears that the IRAP effect is not subject to change by motivational forces such as societal pressures and explicit instructions. For instance, white 
individuals demonstrated pro-white attitudes which were incongruent with their explicit attitudes (Barnes-Holmes et al. 2010a). In addition, even when explicitly instructed on how to fake an IRAP, participants failed to conceal their established verbal relations (McKenna et al. 2007).

The IRAP program requires participants to complete up to three pairs of practice blocks in order to proceed to the fixed set of six test blocks. In these practice blocks, participants must reach the set criteria for that IRAP on a consecutive pair of blocks (e.g., $80 \%$ accuracy and $2000 \mathrm{~ms}$ response latency). One critical issue that often arises in IRAP research is the problem of participants failing to achieve the latency and/or accuracy criteria on the practice blocks, or participants failing to maintain the criteria throughout the six test blocks. When this occurs, the participants' dataset is often removed from the entire experiment. An examination of the IRAP literature shows that studies on average lose approximately $15-22 \%$ of participants through failure to meet or retain criteria. Some studies have seen drop-out rates of up to $50 \%$ due to this problem. Anecdotal evidence suggests that much of participant attrition is likely due to instructional issues in that it appears that experimenters with more experience using the IRAP report far lower attrition rates (i.e., around $10 \%$ ) than those with less experience. A casual review of the IRAP literature indicates that the majority of studies lose participants due to a drop in accuracy rates rather than issues surrounding response latency.

One problem that emerges with participant attrition, of course, is that those participants who fail to meet or maintain the required performance criteria may do so due to reactions to the very stimulus domain that is being targeted in the research. For instance, Nicholson and Barnes-Holmes (2012a) reported that in an IRAP designed to measure spider fear, four participants from the high-fear spider group were removed from the final analysis compared to one from the low-fear group due to a failure to uphold the accuracy criteria. While the aforementioned study yielded positive results, it is possible that results may be skewed toward the non-fearful if one group of participants yields higher levels of attrition relative to another. Occurrences such as this in IRAP research could reduce the likelihood of obtaining a thorough picture of responding in the domain under scrutiny, ultimately masking important results. It is possible that the anxiety elicited by the pictures of spiders used in the study (in particular in the high fear participants) resulted in impaired performance on the IRAP. Gerdes et al. (2008) reported a similar effect which suggests that spider phobic individuals fail to disengage their attention from spider stimuli in a reaction time task. Individuals with contamination-based OCD also demonstrated an inability to disengage from fearful and disgusting stimuli on a reaction time task (Cisler and Olatunji 2010). In relation to the IRAP, it is unclear whether salient stimuli cause the participants' attention to be diverted from the purpose of the task resulting in a greater number of errors or longer response latencies on specific trials only or if this lapse of attention filters throughout the rest of the task also. A theory known as Attentional Control Theory posits that anxiety results in impaired attentional control, which causes poorer performance in tasks which involve working memory, specifically the central executive. $^{1}$ (Coombes et al. 2009). Due to the seemingly attentionally demanding nature of the IRAP, this theory may offer a possible reason for the high attrition rates in IRAP research.

Attentional Control Theory (ACT) was put forth by Eysenck et al. (2007) to explicate the effects of anxiety on cognitive performance. The two main functions within ACT have been identified as the Inhibition function which is the ability to purposefully inhibit prepotent processes when needed and focus on relevant ones; and the Shifting function which is the ability to shift between multiple tasks and mental sets (Miyake et al. 2000; Eysenck et al. 2007). ACT posits that anxiety causes an increase in the salience of the stimulusdriven attentional system and a decrease in the goal-oriented system (Eysenck et al. 2007). Anxiety causes worry about threat to a current goal and anxious individuals come up with strategies to counteract this worry in order to achieve their goal (Eysenck et al. 2007; Derakshan and Eysenck 2009). This could be applicable to the IRAP as participants need to inhibit or disengage from the emotionally-relevant stimuli or from their covert responses to the stimuli (stimulus-driven) and focus on the task at hand (goal-oriented). An inability to do this may result in a greater number of errors and/or slower speed of responding.

Attentionally demanding tasks, such as the IRAP, likely implement the central executive of working memory, and, as a result, performance may be negatively affected by current levels of anxiety. If highly distracting stimuli are presented (e.g., pictures of spiders for spider-fearful individuals) they may, thus, impact on the inhibition and shifting functions of attentional control (Lavie et al. 2004; Eysenck and Derakshan 2011). Evidence supporting this claim has found that highanxious individuals have a greater propensity for distraction than low-anxious individuals (Pacheco-Ungietti et al. 2010). Insofar as the IRAP is demanding on the central executive, and anxiety can impair cognitive performance, it stands to reason that high-anxious individuals doing an IRAP designed to measure anxiety, and its related constructs, may struggle to maintain the necessary accuracy or latency criteria. On balance, high-anxious and low-anxious individuals may demonstrate comparable performances if high-anxious individuals attempt to compensate for performance deficiencies by expending more effort (in the form of greater attentional

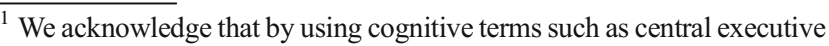
and working memory we are drawing from two separate literatures, however, these terms are ill-defined in behavioral psychology and are the accepted terms in the field of cognitive psychology. Thus, we simply use them here because they are used to investigate the variable of interest.
} 
control) than low-anxious individuals (Eysenck et al. 2007). As an aside, it should be noted that IRAPs that do not use anxiety-provoking stimuli sometimes still report high attrition rates, and, thus, it is unclear if the task itself elicits anxiety which in turn affects performance or if there is another variable which is detrimental to performance.

The current study sought to determine the circumstances under which participants will fail to uphold criteria on the IRAP and if there are any predictive variables that can prevent this from happening. The spider fear IRAP used in Nicholson and Barnes-Holmes (2012a) was employed along with a series of questionnaires such as the Fear of Spiders Questionnaire (FSQ), State-Trait-Anxiety Inventory (STAI) and the Attentional Control Scale (ACS) and a Behavioral Approach Task (BAT) with a live tarantula. Additionally, a symbol version of the $n$-back task, which is assumed to place great demands on working memory and attention because it involves updating, monitoring and manipulating previously remembered information (Chen et al. 2008; Owen et al. 2005), was implemented to determine if performance on an attentionally demanding task is predictive of IRAP performance. The present study was different from previous IRAP studies as it did not make use of the $D$-IRAP scores as the purpose was not to measure spider fear but to determine the variables which predict response latency and accuracy of the IRAP. As such, raw response latencies to specific stimuli and accuracy were the results of interest for this study. It was hypothesized that performance deficits on the $n$-back would be comparable to those on the IRAP. Based on the attentional control literature, it was also hypothesized that scores on the ACS and its sub-components as well as the FSQ and the STAI would be related to accuracy on both tasks. Finally, evidence from the literature suggests that high-anxious individuals have difficulty disengaging from threatening stimuli (Koster et al. 2004), thus, it was assumed that high fear participants would produce longer response latencies on the IRAP than low fear participants.

\section{Method}

Participants

A random sample of 32 undergraduate students from the National University of Ireland, Maynooth volunteered to take part in the current study. Informed consent was obtained from each participant prior to them taking part in the experiment. The sample consisted of 18 women and 14 men, who had a mean age of 21.3 years. There were no exclusion criteria implemented for the study. Each participant completed the study individually in the Department of Psychology at NUI Maynooth.

\section{Materials}

\section{Live Tarantula}

A live tarantula (Brazilian Black) was used throughout the study. It measured approximately $11 \mathrm{~cm}$ and was a pet of a member of staff in the Department of Psychology at NUI Maynooth. The tarantula was confined to a plastic terrarium for the duration of the experimental process.

Fear of Spider Questionnaire (FSQ; Szymanski and O'Donohue 1995)

The FSQ is a self-report scale consisting of 18 items that measures spider fear (e.g., "If I saw a spider now, I would think it would harm me"). Szymanski and O’Donohue (1995) found that phobic and non-phobic individuals yield significantly different scores on the FSQ based on data obtained from 338 undergraduate students. The FSQ has high testretest reliability (.97) and a split-half reliability coefficient of .89 , along with high internal consistency, Cronbach's alpha $=.92$ in a non-clinical sample (Szymanski and O'Donohue 1995). Internal consistency for the present sample was excellent, Cronbach's Alpha $=.96$.

State-Trait Anxiety Inventory-Form Y-1 (STAI; Spielberger et al. 1983)

The State-Trait Anxiety Inventory-Form Y-1 is a 20 item selfreport subscale of the STAI designed to measure state anxiety, i.e., how an individual is feeling right now (e.g. "I feel calm"). Each item is rated on a 5-point $(0-4)$ Likert scale of the level of present anxiety. This subscale of the STAI has been found to have excellent internal consistency among college students, Cronbach's alpha $=.90-.91$ (Spielberger et al. 1983). Internal consistency for the present sample was excellent, Cronbach's Alpha $=.89$.

Attentional Control Scale (ACS; Derryberry and Reed 2002)

The ACS is a self report measure of attentional control, which tests two attentional functions; the inhibition function (the ability to maintain attention for a current task regardless of distracters; e.g., "When I am working hard on something, I still get distracted by events around me") and the shifting function (the ability to switch focus between multiple tasks; e.g., "After being interrupted or distracted, I can easily shift my attention back to what I was doing before"). The questionnaire is composed of 20 items. Individuals are asked to rate their personal response to each item on a 4 point Likert scale $(1=$ almost never, $4=$ always $)$. A total ACS score indicates the individual's ability to control his or her attention. Ólafsson et al. (2011) reported internal consistency for the 
total score and inhibition function as good (Cronbach's Alpha $=.84$ and., 82 respectively). In addition, the shifting function was found to have a lower but still adequate level of internal consistency (Cronbach's Alpha=.68). Internal consistency for the present sample for the overall score was acceptable, Cronbach's Alpha $=.72$, and was also acceptable for the inhibition subscale (Cronbach's Alpha=.72), however, internal consistency was poor for the shifting function subscale (Cronbach's Alpha=.62).

Implicit Relational Assessment Procedure (IRAP; Barnes-Holmes et al. 2006)

The IRAP used during the current study was the same IRAP used in Nicholson and Barnes-Holmes (2012a). Each trial contained either a fear type label ("Frightens Me", "Disgusts Me", "Scares Me", "Creeps Me Out") or an approach type label ("I Could Approach", "I May Approach", "I Can Approach", "I Will Approach"). Simultaneously one of eight target stimuli was presented, which consisted of four color pictures of spiders and four color pictures of landscapes. The pleasant stimuli (i.e., landscapes) were taken from the International Affective Picture System (IAPS; Lang et al. 1996) and the spider images were those used by Huijding and de Jong (2007). In addition, two response options "True" and "False" were displayed on the screen. All stimuli remained present on the screen until a response key was pressed (See Fig. 1). If a response key was not pressed within the time allowed (e.g., $2000 \mathrm{~ms}$ ) the words "Too Slow" appeared on the screen to prompt the participant for an answer. If an incorrect response was given a red " $X$ " appeared on the screen, which remained there until the correct response was made. The IRAP program insured that all eight picture stimuli and all eight word stimuli were presented twice within each block of trials. In addition, the trials were presented quasirandomly, with the constraint that each of the four trial types appeared eight times within each 32-trial block. The program also ensured that the same trial type was not presented across successive trials. Finally, the left-right positioning of the two response options ("True" and "False") alternated randomly across trials with the constraint that they could not appear in the same positions across four successive trials.

Participants completed up to six practice and six test blocks, with each block consisting of 32 trials. Correct responses were dependant on whether the block was termed consistent or inconsistent. Consistent blocks were defined as those that required responses that were in accordance with the beliefs of spider phobic individuals (e.g., selecting "True" when presented with "Frightens $\mathrm{Me}$ " and a spider image; selecting "False" when presented with "I Can Approach" and an image of a spider). Inconsistent blocks were defined as those counter to the beliefs of spider phobics (e.g., selecting "False" when presented with "Frightens Me" and a picture of a spider; selecting "True" when presented with "I Can Approach" and an image of a spider). Participants were told that the IRAP would begin with a consistent block and would alternate between the two block types across the remaining blocks. Participants were informed that, between the

Fig. 1 Examples of the four trialtypes in the IRAP. Note. The boxed words ("Consistent" and "Inconsistent") and the arrows did not appear on screen for participants

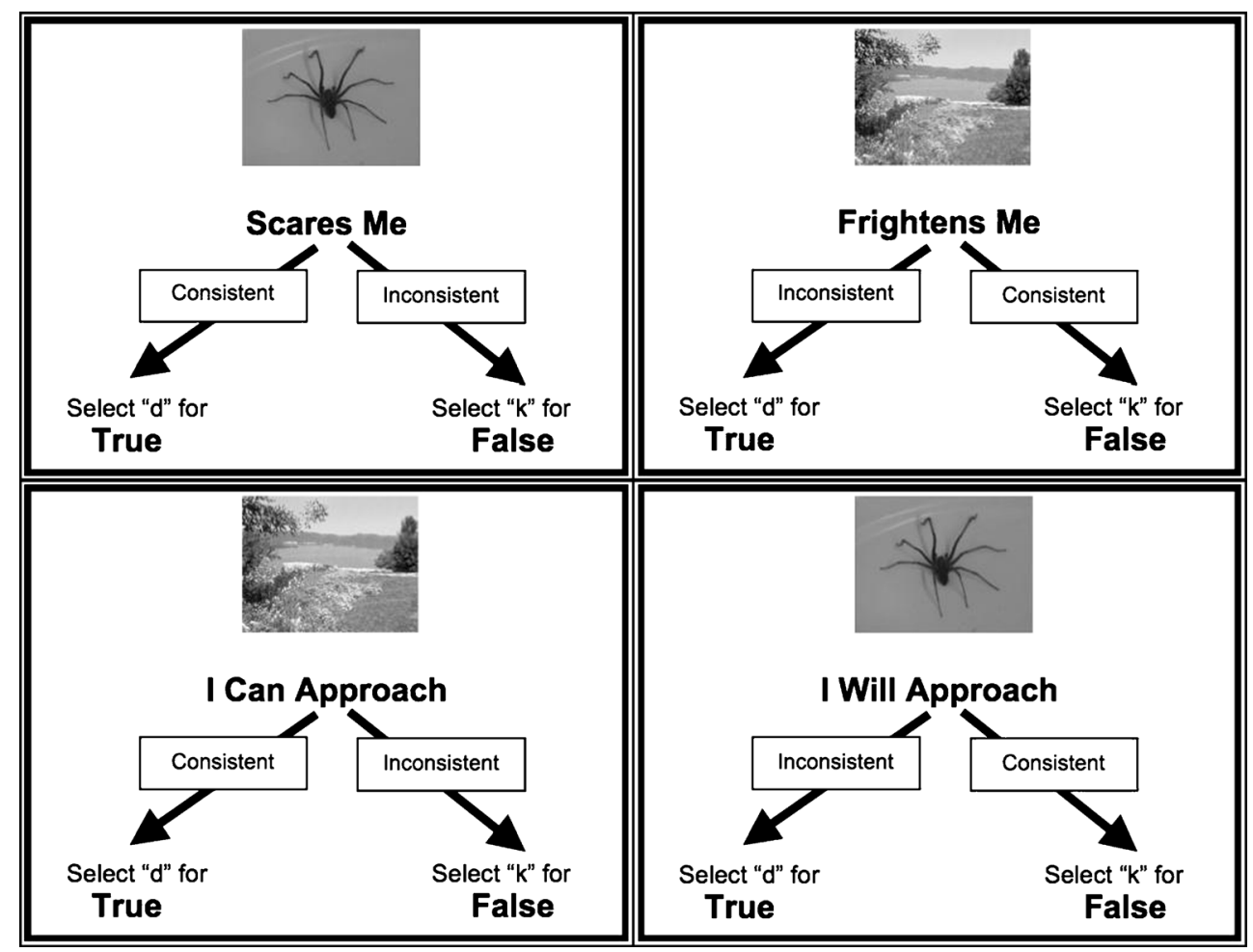


completion of one block and prior to the commencement of another, that all the previous correct answers were now incorrect and vice-versa. Finally, participants were made aware that, at times, they would be required to respond in a manner that was consistent with their own beliefs and, at other times, in a manner that was inconsistent with their beliefs.

$n$-back task

The $n$-back (Gevins and Cutillo 1993) was used as a cognitive task to act as a comparison to the IRAP. The task requires participants to monitor a series of stimuli and to determine on each trial which stimulus was presented $n$ trials previously, where $n$ is a pre-specified number (Owen et al. 2005). The $n$ back used in the present study had a 2-back memory load and four stimulus types could be presented. A rectangle was presented on the screen to each participant, in which one of four symbols (e.g., an asterisk, a square, a triangle or a spider symbol) appeared. They were informed that each symbol corresponded to a key on the keyboard $(1=*, 2=\boldsymbol{\Lambda}, 3=, 4=\boldsymbol{\square})$, which were not marked on the keyboard. Each stimulus was presented for two seconds with only one symbol being presented at any one time. The stimuli changed regardless of the participant's response or non-response. A fixation cross lasting $500 \mathrm{~ms}$ appeared in between each trial. A response was given by pressing the correct key, with keys 1-4 (on a standard keyboard) corresponding to one of the four stimuli. Each participant was informed that their task was to watch the sequence of symbols and to correctly identify the symbol that appeared two symbols before the present one on screen. That is, if the first two trials presented a square followed by a spider and the third trial presented an asterisk, the correct response key to press when the asterisk appeared was the key for the square. Blank trials were presented in pairs throughout the test as a reset. The $n$-back was run using E-prime (Schneider et al. 2002), which administered instructions, stimuli and recorded responses. Each participant completed a practice block of 12 trials and then a test block of 77 trials.

\section{Behavioral Approach Task (BAT; adapted from Nicholson} and Barnes-Holmes 2012a)

Participants were informed that an adjoining room held a live spider in it. They were subsequently asked to approach the spider, which was contained in a terrarium, and were allocated a rating between $0-6$, depending on how close they came to the spider $(0=$ not allowing the experimenter to open the door, $1=$ allowing the experimenter to open the door, $2=$ walking into the room but staying by the door, $3=$ walking up to the terrarium and standing beside it, $4=$ touching the terrarium, $5=$ experimenter lifts up the terrarium and the participant places their hand underneath where the spider is situated and $6=$ the participant holds their hand underneath the terrarium for a full minute). Each participant was awarded a score that reflected how many steps they completed successfully (i.e., 1 for completing step 1, 2 for completing step 2, and so on up to a maximum score of 6).

\section{Mental Effort Scale (Paas 1992)}

The perceived amount of mental effort expended by each participant during the IRAP and during the $n$-back was recorded with the use of a 1 item self report scale. The amount of effort used corresponded to a numerical value between 1-9, $1=$ "very little mental effort" and 9="very high mental effort". The current scale was based on one used by Paas (1992), which has been found to yield a satisfactory reliability coefficient (Cronbach's Alpha .90).

\section{General Procedure}

Participants completed the questionnaires first in the order of STAI, FSQ and ACS. The STAI was completed first to measure state anxiety at the beginning of the experiment. Participants then completed the IRAP, the $n$-back task and the BAT with the order in which the participants completed each task being counterbalanced. The instructions for both the IRAP task and the $n$-back task were presented on paper and were read through with the experimenter to ensure the participant knew what was being asked of them. Participants completed the Mental Effort Scales after both the IRAP and the $n$ back. Finally, at the end of the experiment, participants completed an STAI.

\section{Data Analytic Strategy}

The purpose of the study was to determine the factors which effect accuracy and response latency on the IRAP, and, thus, all analyses were performed using response latencies from each of the stimuli used in the IRAP and $n$-back tasks along with the number or percentage of correct responses. Additionally, the analyses from the questionnaires and performance on the BAT were also included. Due to the primary focus of the study, the $D$-IRAP scores were not analyzed. Pearson product-moment correlations were computed to examine the relationships between the variables. There were no formal procedures implemented to correct for multiple testing due to the small sample size in an effort to avoid making a type 1 error (the actual $p$ values for the correlations are included with the $r$ values to indicate actual levels of significance). Hierarchical multiple regressions were used to determine the factors which predict IRAP performance while controlling for other variables (e.g., pre-experimental anxiety and spider fear). 


\section{Results}

\section{Scoring the IRAP}

The IRAP data contains response latencies and accuracy for each participant, which is central to the scoring process. Typically, these two variables are used to screen out participants by removing those individuals who fail to maintain specific latency and accuracy criteria across the test blocks. Given that the purpose of the current study was to determine if fear-inducing stimuli and anxiety levels impact on the very variables that are typically used to screen out participants, it would be inappropriate to remove those participants in the present research. Three participants, thus, remained within the final sample that would otherwise have been removed (one participant failed to complete the practice blocks and thereby failing to provide any data for analysis). Finally, also due to the focus of the study, both average response latencies and average response accuracy across all of the test blocks for each participant, for the spider and pleasant trial-types, provided the primary data (i.e., the data were not divided into fear "consistent" versus fear "inconsistent" test blocks or transformed into $D$-IRAP scores). Table 1 contains mean values and standard deviations for all measures.

\section{Explicit/Behavioral Correlations}

A correlation matrix was calculated to examine the relationships between the self-report measures (questionnaires and the Mental Effort sales) and avoidance behavior as measured by the BAT (See Table 2). It revealed that pre-experimental anxiety was positively related to spider fear; also there was a significant negative correlation between approach behavior

Table 1 Means and standard deviations from all measures

\begin{tabular}{llll}
\hline & & Mean & SD \\
\hline Accuracy & $n$-back & 54.18 & 15.549 \\
& IRAP & 90.680 & 6.295 \\
$n$-back & Spider & 502 & 295 \\
Response Latency (ms) & Star & 490 & 331 \\
& Triangle & 492 & 356 \\
& Square & 491 & 293 \\
IRAP Response Latency (ms) & Spider & 1339 & 345 \\
& Pleasant & 1351 & 341 \\
STAI Pre-Experiment & & 31.7 & 8.2 \\
FSQ & & 28.8 & 26.5 \\
ACS & & 48.1 & 7.1 \\
STAI Post-Experiment & & 33.1 & 11.4 \\
\hline
\end{tabular}

Note: STAI: State-Trait Anxiety Inventory; FSQ: Fear of Spiders Questionnaire; ACS: Attentional Control Scale and self-reported attentional control. Finally, self-reported spider fear and post-experimental anxiety were negatively related to performance on the BAT. In summary, less avoidance behavior was associated with low spider fear and low levels of state anxiety at the end of the experiment. Interestingly, greater avoidance behavior was related to higher levels of self-reported attentional control suggesting that the spider fearful individuals believed they had good attentional control.

\section{Predicting Accuracy and Response Latency on the IRAP}

\section{Correlational Analysis}

The second set of analyses sought to determine if performance on the IRAP and $n$-back tasks (including accuracy and response latencies on each stimulus) was predicted by spider fear (as measured by the FSQ) avoidance behavior, attentional control and its two constituents (as measured by the ACS), anxiety level at both pre-experimental and post-experimental sequence (as measured by the STAI) or mental effort (as measured by the Mental Effort Scale, with respect to the IRAP and the $n$-back). Additionally, the analyses assessed whether performance on a cognitively demanding task (as measured by the $n$-back), would be predictive of performance on the IRAP. The results of the correlational analyses are presented in Table 3. IRAP accuracy correlated significantly with the ACS (Inhibition) and accuracy on the $n$-back. These results, thus, indicated that greater accuracy on the IRAP predicted higher levels of inhibitory control and perhaps rather predictably higher accuracy on another performance based measure that required attentional control. Interestingly, accuracy on the $n$-back correlated negatively with the FSQ, indicating that greater WM and attention predicted lower levels of self-reported spider fear. Critically, the correlational analyses indicate that neither accuracy nor response latency on the IRAP was influenced significantly by the measures associated with spider fear or anxiety. Thus, it seems unlikely that the data for many participants are removed from IRAP studies based on individual differences in these two domains. Accuracy on the $n$-back was negatively related to response latency on each of the four stimuli used suggesting that faster responding was indicative of greater accuracy. Finally, there were significant positive correlations between scores on the FSQ and response latencies on the spider, star and triangle stimuli along with significant negative correlations between avoidance behavior and response latencies on the star and triangle stimuli.

\section{Regression Analyses}

Three hierarchical multiple regression analyses were conducted to confirm that the Inhibition function predicted IRAP 
Table 2 Pearson Product-moment correlations between the self-report measures and the BAT

\begin{tabular}{|c|c|c|c|c|c|c|c|}
\hline & STAI Pre & FSQ & $\mathrm{ACS}$ & STAI Post & BAT & Mental Effort IRAP & Mental Effort $n$-back \\
\hline STAI Pre & - & $.37 *$ & -.21 & .16 & -.20 & -.1 & -.07 \\
\hline FSQ & & - & .16 & .29 & $-.77 * *$ & -.17 & .28 \\
\hline $\mathrm{ACS}$ & & & - & .15 & $-.37 *$ & .03 & $.34^{*}$ \\
\hline STAI Post & & & & - & $-.35 *$ & .25 & .33 \\
\hline BAT & & & & & - & .20 & -.28 \\
\hline Mental Effort IRAP & & & & & & - & -.03 \\
\hline
\end{tabular}

Note: STAI: State-Trait Anxiety Inventory; FSQ: Fear of Spiders Questionnaire; ACS: Attentional Control Scale; BAT: Behavioral Approach Task $* p<.05$

$* * p<.01$

accuracy after controlling for the effects of pre-experimental anxiety and spider fear (when polynomial regression analyses were conducted, these yielded the same statistical conclusions as those reported subsequently). The first hierarchical multiple regression entered pre-experimental anxiety (pre-STAI) into step 1 of the model, which accounted for $9.2 \%$ of the variance, $\beta=-.30, p=.09$. The Inhibition function was then entered as step 2 of the model and was shown to account for $12.8 \%$ of the variance, $\beta=.36, p=.04$. The next regression analysis, entered FSQ as step 1 in the model, and it accounted for $.6 \%$ of the variance, $\beta=.07, p=.67$. Similarly, the Inhibition function was entered as step 2 and accounted for $12.6 \%$ of the variance, $\beta=.36, p=.05$. In both cases, therefore, Inhibition predicted IRAP accuracy independently of anxiety and spider fear. A final hierarchical regression was completed based on the correlation found between the FSQ and $n$-back accuracy (see Table 1), which sought to confirm that $n$-back

Table 3 Pearson Product-moment correlations between average IRAP accuracy, IRAP response latencies (RL), $n$-back accuracy and $n$-back response latencies (RL) with nine variables, the FSQ, the BAT, the accuracy was a stable predictor of IRAP accuracy regardless of the effects of spider fear. As before, FSQ was entered as step 1 into the model, accounting for $.6 \%$ of the variance, $\beta=.07, p=.68$. The $n$-back accuracy was subsequently entered as step 2 in the model and was found to account for $26.1 \%$ of variance, $\beta=.58, p=.00$, thus, confirming that $n$-back accuracy does indeed predict accuracy on the IRAP independently of self-reported spider fear.

One final hierarchical multiple regression was carried out to determine if scores on the FSQ could predict accuracy on the $n$-back independently of pre-experimental state anxiety. Anxiety (pre-STAI) was entered at step 1 of the model and was a marginally significant predictor of accuracy on the $n$ back accounting for $9 \%$ of the variance, $\beta=-.31, p=.08$. Scores on the FSQ were entered into step 2 of the model accounting for an additional $23.4 \%$ of the variance, $\beta=-.4$, $p=.02$. Thus, the self-reported spider fear was a significant

ACS (Inhibition and Shifting functions), $n$-back Accuracy, the STAI (pre and post) and the Mental Effort Scales (IRAP and $n$-back)

\begin{tabular}{|c|c|c|c|c|c|c|c|c|c|}
\hline & & \multicolumn{3}{|l|}{ IRAP } & \multicolumn{5}{|l|}{$n$-back } \\
\hline & & Accuracy & Spider RL & Pleasant RL & Accuracy & Spider RL & Square RL & Star RL & Triangle RL \\
\hline \multicolumn{2}{|l|}{ FSQ } & .07 & -.14 & -.01 & $-.42 * *$ & $.45^{* *}$ & .30 & $.56^{* *}$ & $.63 * * *$ \\
\hline \multicolumn{2}{|l|}{ BAT } & -.10 & .14 & .11 & .29 & -.34 & -.23 & $-.44 * *$ & $-.41 * *$ \\
\hline \multirow[t]{2}{*}{ ACS } & Inhibition & $.36^{*}$ & -.27 & -.28 & .04 & .07 & -.06 & .19 & .05 \\
\hline & Shifting & .24 & -.24 & -.24 & .07 & -.08 & -.10 & .05 & -.04 \\
\hline \multicolumn{2}{|c|}{$n$-back Accuracy } & $.42 * *$ & -.07 & -.12 & - & $-.55^{* *}$ & $-.42 *$ & $-.61 * * *$ & $-.67 * * *$ \\
\hline \multirow[t]{2}{*}{ STAI } & Pre & -.30 & .21 & .21 & -.14 & .20 & .14 & .29 & .27 \\
\hline & Post & -.26 & .16 & .15 & -.17 & -.05 & -.14 & .02 & .03 \\
\hline \multicolumn{2}{|c|}{ Mental Effort Scale IRAP } & -.29 & .31 & .32 & -.07 & -.22 & -.15 & -.26 & -.21 \\
\hline \multicolumn{2}{|c|}{ Mental Effort Scale N-Back } & .10 & -.07 & -.07 & .04 & -.08 & -.04 & -.04 & -.00 \\
\hline
\end{tabular}

$* p<.05$

$* * p<.01$

$* * * p<.001$

Note: STAI: State-Trait Anxiety Inventory; FSQ: Fear of Spiders Questionnaire; ACS: Attentional Control Scale; RL: Response Latency 
predictor of accuracy on the $n$-back, independently of levels of state anxiety.

\section{Discussion}

The main finding of the current study was that the inhibition function of ACT was a predictor of performance on the IRAP, specifically in regards to accuracy. There was no relationship between the components of attentional control (inhibition and shifting) and performance on the $n$-back task. Factors such as the relevance of the stimuli to the participant's psychopathology or anxiety did not appear to reduce accuracy on the IRAP; however, spider fear was a predictor of accuracy on the $n$ back. Contrary to the hypothesis, there was no variation in response latencies between participants who scored higher on the FSQ on the spider trial-types. Finally, accuracy on the $n$ back task was a significant predictor of accuracy on the IRAP.

The results are promising regarding the use of the IRAP in clinically relevant domains as it appears that the salience of the stimuli to the psychopathology of the participant did not affect performance on the IRAP. Critically, following an examination of response latencies of both the spider and pleasant trialtypes in the correlation analyses, it did not appear that the higher fear participants produced longer response latencies on the spider trial-types suggesting that they did not struggle to disengage from the spider stimuli as hypothesized. Thus, the results support the use of the IRAP in applied domains as they suggest that clinical or high-anxious participants will not struggle to maintain criteria across the IRAP, more so than a non-clinical sample. It should be noted that the present study was limited as it used a non-clinical student sample that scored across a continuum from low to high fear on the self-report measure. Thus, the high fear participants are only high scoring in comparison to a non-clinical sample. Further research could utilize a clinical sample of spider phobic individuals (or those suffering with a different psychopathology) to determine if there is an effect of the stimuli used in the IRAP in clinical samples, however, the present results are promising.

Correlational analyses revealed that those who demonstrated greater avoidance behavior reported higher levels of spider fear at the beginning of the experiment and greater state anxiety at the conclusion of the experiment. This analysis also produced an interesting finding which showed that those who were highly avoidant on the BAT reported a greater propensity for attentional control. This result conflicts with evidence from the $n$-back task in which higher spider fear appeared to result in a greater number of errors suggesting poorer attentional control. It is possible that there were self-presentational biases at work on the ACS or perhaps the high fear participants were merely unaware of their actual ability to control their attentional resources. Nevertheless, it appears from the present results that high spider fear can impair performance on an attentionally demanding task (i.e., the $n$-back) in which spider stimuli are presented. The current results are not the first to show the effects of psychopathology on the $n$-back task. For example, previous research found that depressed patients demonstrated significant impairments in performance on an $n$-back task compared to controls (Harvey et al. 2004). Regarding response latency on the $n$-back, high levels of spider fear were related to longer response latencies on the spider, star and triangle stimuli. A possible explanation of this effect could be due to having to respond to the other stimuli while the spider is on the screen or having to respond to the spider while the other stimuli were on the screen. Thus, there is a greater interaction between the stimuli rather than on the IRAP. Overall, this finding is consistent with Attentional Control Theory as it posits that anxiety (likely evoked from the spider stimuli) results in impaired attentional control on a task such as the n-back that implements working memory (Eysenck et al. 2007; Derakshan and Eysenck 2009).

The current findings suggest that differences in a specific cognitive ability, the inhibition function, are responsible for the participants' ability to achieve and maintain performance criteria across the test blocks of the IRAP. Normally, the $D$ IRAP transformation controls, at least to some extent, for differences in cognitive ability across participants, and ,thus if the performance criteria are met and maintained, individual differences in cognitive ability becomes largely irrelevant during the final analysis. However, participants are typically eliminated from studies before a $D$-score is calculated because they do not maintain criteria across the test blocks. The current findings suggest that the inhibition subscale of the ACS may provide a way to screen participants to determine who may need extra assistance in getting through the IRAP. Of course, should participants be screened before completing the IRAP, it is important to implement valid methodologies which will assist them in achieving the necessary criteria.

The purpose of the inhibition function is to refrain from attending to task-irrelevant stimuli and responses (Eysenck and Derakshan 2011), and as mentioned previously, anxiety impairs performance of the inhibition function (see Derakshan and Eysenck 2009). Thus, it could be argued that anxiety has a detrimental effect on the inhibition function, which results in a greater number of mistakes made on the IRAP. However, the source of this anxiety remains unclear. The present results suggest that self-reported spider fear and preexperimental anxiety were not predictive of lower accuracy on the IRAP, however, the task itself may be eliciting anxiety in the participants. Indeed, there was a slight increase in state anxiety by the end of the experiment. However, future research could further examine the possibility that test anxiety adversely impacts IRAP performance through the inhibition function as previous research has found that test anxiety negatively effects achievement (Hembree 1988). 
Previous research has utilized a practice IRAP to familiarize participants with the task before doing the IRAP proper (Vahey et al. 2010). One issue around this method is that a practice IRAP can be time-consuming and more importantly participants may be cognitively "tired" by the time the second IRAP is presented, which could result in more errors. Insofar as differences in attentional control appear to predict variations in accuracy, a method which improves this factor could be highly beneficial. Some researchers have posited that mindfulness, which is enhanced attentiveness and awareness of present experience (Brown and Ryan 2003), possesses many features of attentional control. Indeed, poor attentional control is indicative of low levels of mindfulness (Baer et al. 2006; Walsh et al. 2009), thus, it is possible that implementing a mindfulness task before completion of the IRAP may help participants to avoid attending to task-irrelevant thoughts and feelings that may arise while carrying out the IRAP. However, further research is vital to determine the intricacies that would be necessary in order for the mindfulness task to improve attentional control in the relatively short duration of an experiment.

One limitation of the current study is the relatively small sample size, and, thus, the absence of statistically significant effects might be due, in part, to insufficient power. Of course, future studies that employ the IRAP in "pure" social or clinical research that would be difficult and/or too costly to replicate should employ larger samples to control for random statistical aberrations. A second possible critique is the inclusion of landscapes as the opposing category in the IRAP because the concept "I could approach a landscape" may have been confusing for participants. Spiders have always been a difficult category to use in the IRAP and other implicit measures due to the lack of a natural opposite and this has been widely documented in the literature (see Teachman 2007; Nicholson and Barnes-Holmes 2012a). However, the use of other animals such as dogs or cats as opposing categories can also be problematic; for instance, a picture of a puppy or a kitten may be negatively valenced for some individuals due to specific allergies to domestic pets.

It is worth noting that accuracy on the IRAP was positively related to accuracy on the $n$-back task. That is, those that made a greater number of errors in responding on the $n$-back also made a higher number of errors on the IRAP. The $n$-back task is a test of working memory and attention switching (Sliwinski et al. 2006), which provides empirical evidence of the assumption that working memory is required while carrying out the IRAP. However, high levels of self-reported spider fear (as measured by the FSQ) were related to a greater number of errors on the $n$-back task, whereas self-reported spider fear did not influence performance on the IRAP. Thus, it appears that the cause of errors on the IRAP is different from that of the $n$-back. Namely, the $n$-back may be more affected by the relevance of the stimuli to the psychopathology of the participant. This could also be due to procedural differences between the tasks. For example, the IRAP employed in the current study presented up to six thirty-two trial practice blocks while the $n$-back presented a total of twelve practice trials. Thus, the participants may have become desensitized to the anxiety evoked by the spider pictures on the IRAP before the test blocks, which resulted in less distraction whereas the relatively limited practice for the $n$-back may not have allowed the fear to be extinguished before the test block. This result suggests that not only are the practice blocks useful for familiarizing participants with the IRAP task, they also may serve as a way in which to extinguish internal responses which may be detrimental to performance on the IRAP. Critically, however, any such desensitization effects do not appear to undermine the validity of the IRAP as a measure because previous IRAP research has demonstrated between-group differences across a range of domains relevant to psychopathology, including fear (Nicholson and BarnesHolmes 2012a), addiction (Carpenter et al. 2012), depression (Hussey and Barnes-Holmes 2014) and disgust (Nicholson and Barnes-Holmes 2012b).

Acknowledgements The preparation for this article was supported by the John and Pat Hume Scholarship Scheme and the Irish Research Council. Correspondence concerning this article should be sent to Emma Nicholson (email: Emma.Nicholson@nuim.ie)

\section{References}

Baer, R., Smith, G., Hopkins, J., Krietemeyer, J., \& Toney, L. (2006). Using self-report assessment methods to explore facets of mindfulness. Assessment, 13, 27-45. doi:10.1177/1073191105283504.

Barnes-Holmes, D., Barnes-Holmes, Y., Power, P., Hayden, E., Milne, R., \& Stewart, I. (2006). Do you really know what you believe? Developing the Implicit Relational Assessment Procedure (IRAP) as a direct measure of implicit beliefs. The Irish Psychologist, 32, $169-177$.

Barnes-Holmes, D., Barnes-Holmes, Y., Stewart, I., \& Boles, S. (2010a). A sketch of the Implicit Relational Assessment Procedure (IRAP) and the Relational Elaboration and Coherence (REC) model. The Psychological Record, 60, 527-542.

Barnes-Holmes, D., Murtagh, L., Barnes-Holmes, Y., \& Stewart, I. (2010b). Using the Implicit Association Test and the Implicit Relational Assessment Procedure to measure attitudes toward meat and vegetables in vegetarians and meat-eaters. The Psychological Record, 60, 287-305.

Brown, K. W., \& Ryan, R. M. (2003). The benefits of being present: Mindfulness and its role in well-being. Journal of Personality and Social Psychology, 84, 822-848. doi:10.1037/0022-3514.84.4.822.

Carpenter, K. M., Martinez, D., Vadhan, N. P., Barnes-Holmes, D., \& Nunes, E. V. (2012). Measures of Attentional Bias and Relational Responding are Associated with Behavioral Treatment Outcome for Cocaine Dependence. The American Journal of Drug and Alcohol Abuse, 38, 146-154. doi:10.3109/00952990.2011.643986.

Chen, Y.-N., Mitra, S., \& Schlaghecken, F. (2008). Sub-processes of working memory in the N-back task: An investigation using ERPs. Clinical Neurophysiology, 119, 1546-1559. 
Cisler, J. M., \& Olatunji, B. O. (2010). Components of attentional biases in contamination fear: Evidence for difficulty in disengagement. Behaviour Research and Therapy, 48, 74-78. doi:10.1016/j.brat. 2009.09.003.

Coombes, S. A., Higgins, T., Gamble, K. M., Cauraugh, J. H., \& Janelle, C. M. (2009). Attentional Control Theory: Anxiety, emotion, and motor planning. Journal of Anxiety Disorders, 23, 1072-1079. doi: 10.1016/j.janxdis.2009.07.009.

Dawson, D. L., Barnes-Holmes, D., Gresswell, D. M., Hart, A. J. P., \& Gore, N. J. (2009). Assessing the implicit beliefs of sexual offenders using the Implicit Relational Assessment Procedure: A First Study. Sexual Abuse: A Journal of Research and Treatment, 21, 57-75. doi: 10.1177/1079063208326928.

Derakshan, N., \& Eysenck, M. W. (2009). Anxiety, Processing Efficiency, and Cognitive Performance. European Psychologist, 14, 168-176. doi:10.1027/1016-9040.14.2.168.

Derryberry, D., \& Reed, M. A. (2002). Anxiety related Attentional Biases and their Regulation by Attentional Control. Journal of Abnormal Psychology, 111, 225-236. doi:10.1037//0021-843X.111.2.225.

Eysenck, M. W., \& Derakshan, N. (2011). New perspectives in attentional control theory. Personality and Individual Differences, 50, 955-960. doi:10.1016/j.paid.2010.08.019.

Eysenck, M. W., Derakshan, N., Santos, R., \& Calvo, M. G. (2007). Anxiety and Cognitive Performance: Attentional Control Theory. Emotion, 7, 336-353. doi:10.1037/1528-3542.7.2.336.

Gerdes, A. B. M., Alpers, G. W., \& Pauli, P. (2008). When spider appear suddenly: Spider-phobic patients are distracted by spider irrelevant spiders. Behaviour Research and Therapy, 46, 174-187. doi:10. 1016/j.brat.2007.10.010

Gevins, A. S., \& Cutillo, B. C. (1993). Neuroelectric evidence for distributed processing in human working memory. Electroencephalography \& Clinical Neurophysiology, 87, 128143. doi:10.1037/0894-4105.19.6.814.

Harvey, P. O., Le Bastard, G., Pochon, J. B., Levy, R., Allilaire, J. F., Dubois, B., \& Fossati, P. (2004). Executive functions and updating of the contents of working memory in unipolar depression. Journal of Psychiatric Research, 38, 567-576. doi:10.1016/j.neuroimage. 2005.02.048

Hayes, S. C., Barnes-Holmes, D., \& Roche, B. (2001). Relational Frame Theory: A post- Skinnerian account of human language and cognition. New York: Kluwer/Plenum.

Hembree, R. (1988). Correlates, causes, effects, and treatment of test anxiety. Review of Educational Research, 58, 47-77.

Hughes, S., Barnes-Holmes, D., \& De Houwer, J. (2011). The Dominance of Associative Theorising in Implicit Attitude Research: Propositional and Behavioral Alternatives. The Psychological Record, 61, 465-496.

Huijding, J., \& de Jong, P. J. (2007). Beyond fear and disgust: The role of (automatic) contamination-related associations in spider phobia. Journal of Behavior Therapy and Experimental Psychiatry, 38, 200-211. doi:10.1016/j.jbtep.2006.10.009.

Hussey, I., \& Barnes-Holmes, D. (2012). The IRAP as a measure of implicit depression and the role of psychological flexibility. Cognitive and Behavioral Practice, 19, 573-582.

Koster, E. H. W., Crombez, G., Verscheure, B., \& De Houwer, J. (2004). Selective attention to threat in the dot-probe paradigm: differentiating vigilance and difficulty to disengage. Behaviour Research and Therapy, 42, 1183-1192. doi:10.1016/j.brat.2003.08.001.

Lang, P. J., Bradley, M. M., \& Cuthbert, B. N. (1996). International Affective Picture System (IAPS): Technical manual and affective ratings. NIMH Center for the Study of Emotion and Attention.
Lavie, N., Hirst, A., de Fockert, J. W., \& Viding, E. (2004). Load theory of selective attention and cognitive control. Journal of Experimental Psychology: General, 133, 339-354. doi:10.1037/0096-3445.133.3.339.

McKenna, I. M., Barnes-Holmes, D., Barnes-Holmes, Y., \& Stewart, I. (2007). Testing the fake-ability of the Implicit Relational Assessment Procedure (IRAP): The first study. International Journal of Psychology and Psychological Therapy, 7, 253-268.

Miyake, A., Friedman, N. P., Emerson, M. J., Witzki, A. H., Howerter, A., \& Wager, T. D. (2000). The Unity and Diversity of Executive Functions and Their Contributions to Complex "Frontal Lobe" Tasks: A Latent Variable Analysis. Cognitive Psychology, 41, 49100. doi:10.1006/cogp.1999.0734.

Nicholson, E., \& Barnes-Holmes, D. (2012a). The Implicit Relational Assessment Procedure (IRAP) as a Measure of Spider Fear. The Psychological Record, 62, 263-278.

Nicholson, E., \& Barnes-Holmes, D. (2012b). Developing an implicit measure of disgust propensity and disgust sensitivity: Examining the role of implicit disgust propensity and sensitivity in obsessive compulsive tendencies. Journal of Behavior Therapy and Experimental Psychiatry, 43, 922-930. doi:10.1016/j.jbtep.2012.02.001.

Ólafsson, R. P., Smári, J., Guðmundsdóttir, F., Olafsdóttir, G., Harðardóttir, H. L., \& Einarsson, S. M. (2011). Self-reported attentional control with the Attentional Control Scale: Factor structure and relationship with symptoms of anxiety and depression. Journal of Anxiety Disorders, 25, 777-782. doi:10.1016/j.janxdis.2011.03.013.

Owen, A. M., McMillan, K. M., Laird, A. R., \& Bullmore, E. (2005). Nback working memory paradigm: a meta-analysis of normative functional neuroimaging studies. Human Brain Mapping, 25, 4659. doi:10.1002/hbm.20131.

Paas, F. G. W. C. (1992). Training strategies for attaining transfer of problem-solving skill in statistics: A cognitive load approach. Journal of Educational Psychology, 84, 429-434.

Pacheco-Ungietti, A. P., Acosta, A., Callejas, A., \& Lupianez, J. (2010). Attention and anxiety: Different attentional functioning under state and trait anxiety. Psychological Science, 21, 298-304.

Schneider, W., Eschmann, A., \& Zuccolotto, A. (2002). E-Prime reference guide. Pittsburgh, PA: Psychology Software Tools.

Sliwinski, M. J., Smyth, J. M., Hofer, S. M., \& Stawski, R. M. (2006). Intraindividual coupling of daily stress and cognition. Psychology and Aging, 21, 545-557. doi:10.1037/0882-7974.21.3.545.

Spielberger, C. D., Gorsuch, R. L., Lushene, R., Vagg, P. R., \& Jacobs, G. A. (1983). Manual for the state-trait anxiety inventory. Palo Alto, CA: Consulting Psychologists Press.

Szymanski, J., \& O’Donohue, W. (1995). Fear of Spiders Questionnaire. Journal of Behavior Therapy and Experimental Psychiatry, 26, 31-34.

Teachman, B. A. (2007). Evaluating implicit spider fear associations using the Go/No-go Association Task. Journal of Behaviour Therapy and Experimental Psychiatry, 38, 156-167. doi:10.1016/j.jbtep.2006.10.006.

Vahey, N. A., Barnes-Holmes, D., Barnes-Holmes, Y., \& Stewart, I. (2009). A first test of the Implicit Relational Assessment Procedure as a measure of self-esteem: Irish prisoner groups and university students. The Psychological Record, 59, 371-388.

Vahey, N., Boles, S., \& Barnes-Holmes, D. (2010). Measuring adolescent's smoking related social identity preferences with the Implicit Relational Assessment Procedure (IRAP) for the first time: A starting point that explains later IRAP evolutions. International Journal of Psychology and Psychological Therapy, 10, 453-474.

Walsh, J. J., Balint, M. G., Smolira SJ, D. R., Fredericksen, L. K., \& Madsen, S. (2009). Predicting individual differences in mindfulness: The role of trait anxiety, attachment anxiety and attentional control. Personality and Individual Differences, 46, 94-99. doi:10.1016/j. paid.2008.09.008. 